\title{
Erratum to: Estimating extreme quantiles under random truncation
}

Laurent Gardes ${ }^{1}$ • Gilles Stupfler ${ }^{2}$

\section{Erratum to: TEST DOI 10.1007/s11749-014-0403-5}

We would like to point out the following correction to the original article: on the third page, three lines below the definition of $\widehat{\Lambda}_{N}^{F}(y), \widehat{\bar{F}}_{N}(y)$ should be defined as

$$
\widehat{\bar{F}}_{N}(y)=1-\exp \left(-\widehat{\Lambda}_{N}^{F}(y)\right)
$$

The online version of the original article can be found under doi:10.1007/s11749-014-0403-5.

$\bowtie \quad$ Laurent Gardes

gardes@unistra.fr

1 Université de Strasbourg and CNRS, IRMA, UMR 7501, 7 rue René Descartes, 67084 Strasbourg Cedex, France

2 Aix Marseille Université, CNRS, EHESS, Centrale Marseille, GREQAM UMR 7316, 13002 Marseille, France 